\title{
DLR MiroSurge: a versatile system for research in endoscopic telesurgery
}

\author{
Ulrich Hagn · R. Konietschke • A. Tobergte • M. Nickl • S. Jörg • B. Kübler • \\ G. Passig · M. Gröger · F. Fröhlich · U. Seibold · L. Le-Tien · A. Albu-Schäffer • \\ A. Nothhelfer · F. Hacker • M. Grebenstein - G. Hirzinger
}

Received: 17 December 2008 / Accepted: 21 May 2009

(C) CARS 2009

\begin{abstract}
Purpose Research on surgical robotics demands systems for evaluating scientific approaches. Such systems can be divided into dedicated and versatile systems. Dedicated systems are designed for a single surgical task or technique, whereas versatile systems are designed to be expandable and useful in multiple surgical applications. Versatile systems are often based on industrial robots, though, and because of this, are hardly suitable for close contact with humans.

Method To achieve a high degree of versatility the Miro robotic surgery platform (MRSP) consists of versatile components, dedicated front-ends towards surgery and configurable interfaces for the surgeon.

Results This paper presents MiroSurge, a configuration of the MRSP that allows for bimanual endoscopic telesurgery with force feedback.

Conclusions While the components of the MiroSurge system are shown to fulfil the rigid design requirements for robotic telesurgery with force feedback, the system remains versatile, which is supposed to be a key issue for the further development and optimisation.
\end{abstract}

Keywords Telerobotics · Versatility · Force feedback · 3D vision $\cdot$ Lightweight

A short version of this manuscript has been presented at the CURAC.08 conference in Leipzig, Germany [1].

U. Hagn $(\varangle) \cdot$ R. Konietschke · A. Tobergte · M. Nickl · S. Jörg ·

B. Kübler · G. Passig · M. Gröger · F. Fröhlich · U. Seibold

L. Le-Tien - A. Albu-Schäffer - A. Nothhelfer · F. Hacker .

M. Grebenstein · G. Hirzinger

Institute of Robotics and Mechatronics,

DLR, German Aerospace Center, Oberpfaffenhofen, Germany

e-mail: Ulrich.Hagn@dlr.de

URL: http://www.dlr.de/rm/en

\section{Introduction}

The developers of surgical robot systems are following a couple of different strategies. One is to build dedicated machines for specific applications or diseases, such as endoscopic surgery [2] or prostate cancer [3]. In this case, clear requirements enable concrete system specifications and an efficient system design.

In contrast, the idea of versatile surgical systems is different. Flexible robots are part of the operating room facilities and assist surgeons in a wide range of applications. This vision of a versatile robotic surgery assistant lacks clear requirements, since the range of applications is intentionally wide [4].

Industrial robots are suitable for addressing heterogeneous surgical applications [5]. However, today's industrial robots are commonly considered unsuitable for close human contact. Surgical robots should be lightweight, compliant and compact to enable close interaction between clinician, patient and robot.

The approach presented in this paper focuses on establishing a versatile platform, explicit for surgical applications. In this context the scope of versatility comprises

- Multiple surgical domains (e.g. visceral surgery, orthopaedic surgery, neurosurgery)

- Adaptable robotic setups (e.g. varied mounting locations and number of robots)

- Different control modes (e.g. position control, compliance control, admittance control)

- Different types of interaction (e.g. hands-on robotics, telerobotics with force feedback)

- Convenient integration of supplemental technologies (e.g. visual servoing, augmentation)

However, the concept of a versatile robotic system is not precise, thus making it difficult to clearly define system 
requirements. To find an adequate set of requirements, the designers focused on four dedicated applications, which address a challenging range of demands:

- Placement of pedicle screws [6], which includes drilling and milling with vibration damping [7].

- Navigated neurosurgical biopsies, which require precise positioning based on navigation and tracking [8].

- Robot assisted laser osteotomies, which demand coupling with passive kinematics (mirror arm).

- Endoscopic telesurgery, which needs low latency, high dynamics, multiple robot integration and force feedback control methods.

The Miro robotic surgery platform (MRSP) is a configurable and extensible platform that is expected to meet the dedicated demands of various surgical applications. The software and hardware components are integrated as building blocks in a rapid prototyping infrastructure. Ample communication bandwidth and extensible computation power are used to avoid bottlenecks. A flexible tool interface enables the use of various instruments, allowing the system to adapt to varied surgical tasks and techniques.

The intention of this paper is to present the versatility of the MRSP by a dedicated configuration (MiroSurge), which is targeted at minimally invasive robotic surgery (MIRS) of the heart and the abdomen with a telerobotic approach (see Figs. 1, 2).

In MIRS, slender instruments are introduced into the patient's body through small incisions. Robotics is applied to increase the surgeon's effective dexterity and immersion as well as to enable new instrument designs.

The following sections give an overview of the MRSP. The DLR MIRO robot arm and DLR MICA instrument sections describe the main hardware components of the platform. Surgical workstation explains the surgeon's interface. Planning

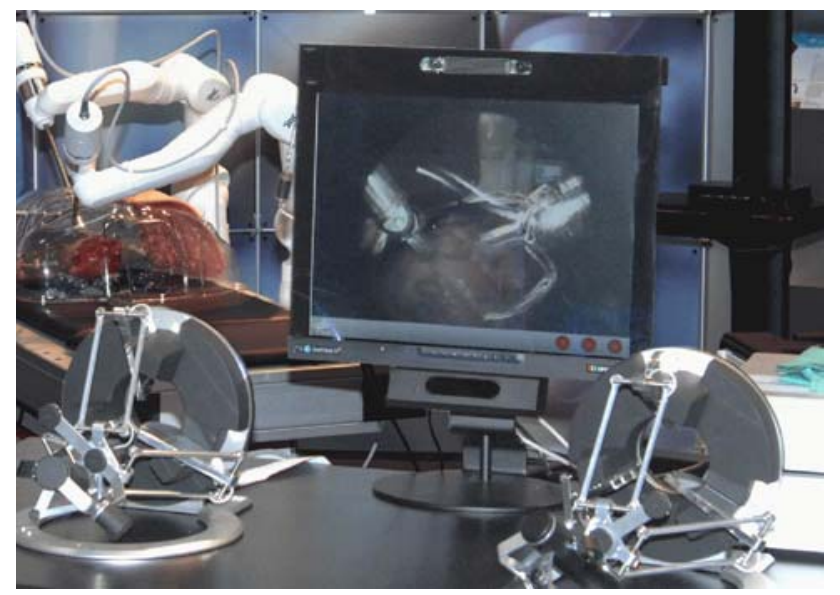

Fig. 1 The DLR MiroSurge user interface

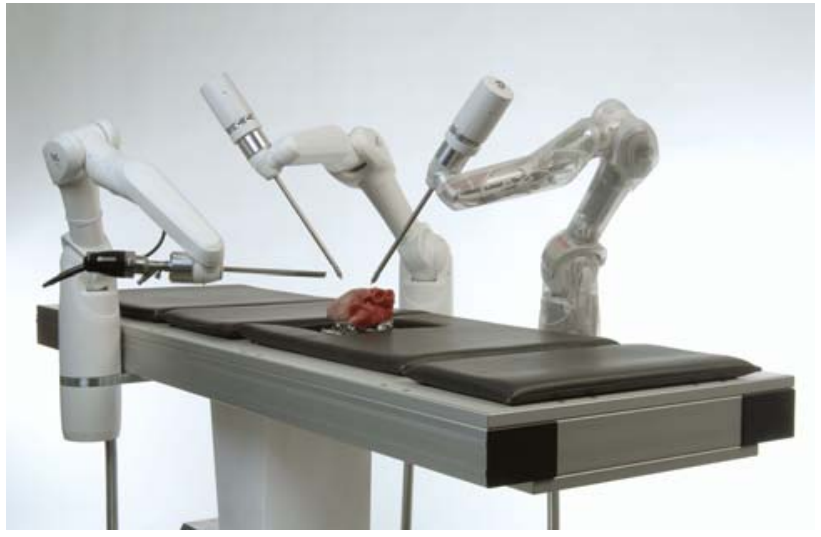

Fig. 2 The DLR MiroSurge robotic system

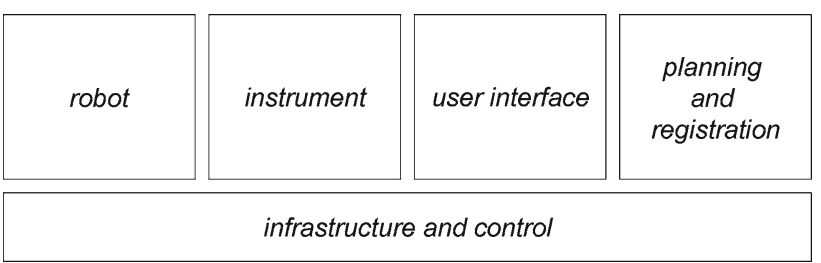

Fig. 3 The partitioning of the DLR MiroSurge robotic system

and registration describes the process for generating optimised robotic setups. Control of the telerobotic system gives an overview of the control implementation and depicts the flexibility of the rapid prototyping software infrastructure.

\section{The Miro robotic surgery platform}

A robotic system for medical applications can be partitioned as shown in Fig. 3. Components can be integrated in one or multiple instances. For the development of the MRSP, the following attributes have been assigned to the components in order to achieve reusable subsystems, convenient configuration, and a satisfying performance:

- The robot is specified as a versatile component since it fulfils the requirements of a broad range of surgical applications. As a platform it enables the implementation of different control modes and is targeted at the extracorporeal manipulation of instruments.

- The specialised instruments are the system's front-end interface towards surgery and specialise the system for dedicated tasks and techniques. They cover all requirements (e.g. additional articulation, sensor integration) that cannot be provided by the robot.

- The user interface comprises all components and signal interfaces for the interaction between the surgeon and the system. In the MiroSurge system, various options can be 
chosen to adapt the system to individual surgeon preferences.

- Planning and registration comprises all methods and components that relate the robotic system and planned data to the current situation in the operating room. In MiroSurge, the focus is on providing convenient methods for optimising the robotic setup and, thus, increasing performance and safety.

- Infrastructure and control interconnects all components. In the MiroSurge system, this component is targeted at high performance, modularity and rapid prototyping capabilities.

The following sections more thoroughly describe the previously listed components of the MiroSurge system.

\section{DLR MIRO robot arm}

One central component of the DLR MiroSurge system is the MIRO robot [9], the second generation DLR robot arm for medical applications, which follows the first generation arm, KineMedic [8]. The central aim of the MIRO robot is to provide a versatile lightweight manipulator for the extracorporeal guidance of instruments in open and minimally invasive surgery. The adaptation of the MIRO robot to a specific surgical application is achieved by adding specialised instruments. Moreover, integrated multi-modal sensors and different control modes allow system configurations for telepresence, autonomous applications, hands-on applications, and combinations of these configurations. The hands-on approach, for example, can be used in a telepresence application by simply exerting forces on the robot's structure to guide the robot during instrument changes.

Interactions with technical systems demand an understanding of the system. Therefore, one important issue for enhancing the acceptance of a system is an inherent predictability of the robot's actions. With the DLR MIRO, this issue is addressed by a serial kinematics which resembles that of the human arm. There is a dedicated shoulder (intersecting roll, pitch, and yaw axes), upper arm, elbow (intersecting pitch, roll axes), forearm and wrist (intersecting pitch roll axes) as depicted in Fig. 4. The joint ranges and the ratio between upper arm and forearm length have been optimised based on the different selected medical procedures [10]. Only the wrist kinematics differs from that of humans due to the frequent rotation of endoscopic instruments about their shaft axes. The redundancy achieved by integrating seven joints is utilised in minimally invasive surgery to enable four degree of freedom (DoF) extracorporeal manipulability, to comply with the two DoF constraint on the fulcrum point and at the same time to establish additional methods for avoiding collisions between robots.

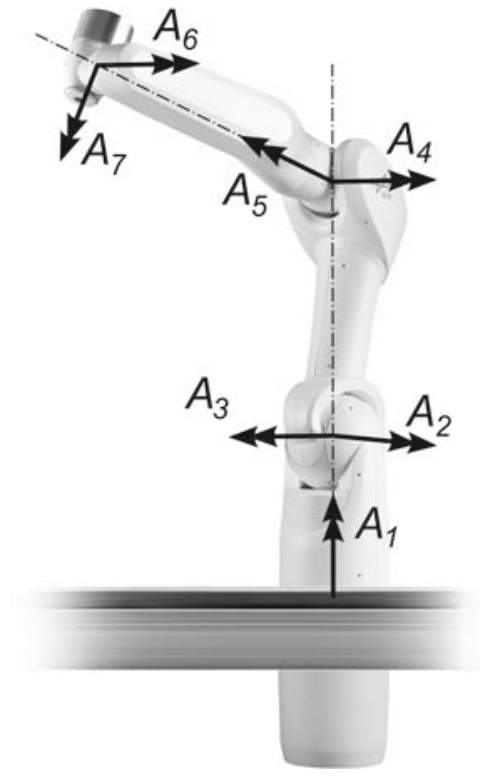

Fig. 4 The DLR MIRO lightweight robot with depicted joint axes

One central issue raised by endoscopic surgical techniques is the kinematic constraint introduced by the fulcrum point, which limits the motion of the endoscopic instrument to four DoFs. One method for addressing this constraint is to use remote-center-of-motion (RCM) manipulators, such as those used in the da Vinci ${ }^{\circledR}$ Surgical System (Intuitive Surgical ${ }^{\circledR}$ Inc.), with a fixed position of the fulcrum point in relation to the manipulator base. The DLR MIRO, in contrast, addresses this constraint by configuring the nullspace of the redundant kinematics and applying position and force control methods. Unlike a dedicated solution, such as RCM manipulators, this solution allows the DLR MIRO to arbitrarily define the position of the fulcrum point within the workspace of the robot, thus allowing for a moving fulcrum point. This is useful for applications that involve a moving entry point, such as the chest wall during respiration. This aspect of the MIRO robot increases the system's flexibility.

The robot arm integrates joint-sided torque and position sensors, motor-sided safety brakes, communication devices and sensor and actuator electronics, thereby reducing the external components to an off-the-shelf PC, a PCIe-Board and a DC power supply. The basic specifications of the robot are shown in Table 1.

To integrate the MIRO robot into the confined environment of an operating room, the design of the robot is aimed at compact dimensions, which allow multiple robots to be set up directly at the operating table as depicted in Fig. 2. Due to the robot's low weight of $10 \mathrm{~kg}$, alternative setups, including ceiling-mounted [11] and wall-mounted systems, are feasible. Therefore, the MIRO robotic system adapts to the operating room and not vice versa. 
Table 1 Specifications of the DLR MIRO robot

\begin{tabular}{ll}
\hline Parameter & Value \\
\hline Number of joints & 7 \\
Mass & $10 \mathrm{~kg}$ \\
Maximum payload & $3 \mathrm{~kg}$ \\
Length $\left(\overline{A_{2} A_{4}}+\overline{A_{4} A_{6}}\right)$ & $0.76 \mathrm{~m}$ \\
Position sensors & Motor-sided, joint-sided \\
Cartesian speed & $0.5 \mathrm{~m} / \mathrm{sec}$ \\
Torque Sensors & Joint-sided \\
Safety brakes & Motor-sided \\
Control modes & Position, torque, and impedance \\
Control cycle & $3 \mathrm{kHz}$ \\
\hline
\end{tabular}

The close contact between robot arm, patient and clinician demands safety features to avoid collisions and reduce their severity if they do occur. With the MiroSurge system, collisions between the robot's end-effectors are avoided by workspace limitations based on exact knowledge of the robots' setup positions and joint angles. Collisions of the robot structures (elbows) are avoided by defining a nullspace criterion which attracts the elbows to distant points. However, this approach only respects objects that are known or detected. In the random and changing environment of an operating room, the feasibility of detecting all objects or personnel near the robots is questionable. Therefore, the MIRO is designed in a way that reduces the severity of unintended collisions that are not avoided through planning or nullspace motions. This issue has been addressed by using low-inertia components and compliant polymer housings. The reflected motor inertia is reduced by torque control up to a factor of five compared to position control, while the link inertia is low due to the lightweight design (e.g. $1.7 \mathrm{~kg}$ forearm and wrist). Additionally, integrated joint torque sensors can monitor the interaction forces between the patient and the robot/instrument and enable the planned implementation of sensitive collision detection as introduced in [12]. Patient safety has various aspects with impact to all system components: reliable surgeon-robot cooperation, robust feedback control and deterministic hardware behaviour. The Miro-Platform provides redundant sensors (e.g. motor and joint position sensors) to detect hardware failures and system-wide emergency stop. In case of malfunctions which would lead to undeterministic behaviour, a dedicated failsafe emergency stop backbone on hardware level turns all actuators off and safety brakes lock the joints. Thus, in case of an error, all robots return to a predictable, safe state that inhibits patient trauma.

However, the communication and computing hardware remains powered to identify errors and to enable fallback solutions. Errors that do not lead to an undeterministic behaviour (e.g. sensorised collisions with other objects) are processed on a software level of the robot control. Robust feedback control is guaranteed by our passivity-based control framework [13]. The aim of passivity-based control is that energy supplied to the system is exactly controlled such that each controller component has an intuitive interpretation as a passive physical device (e.g. virtual spring, damper, mass). This ensures the stability of the system (since the energy of a passive system cannot increase) and the system is very robust with respect to parameter uncertainties and unknown, but passive environmental dynamics.

Endoscopic instruments can be portioned into a functional tip (grippers, scissors, optics, etc.), a long shaft and an extracorporeal drive unit (see Fig. 6). For safety reasons it is important that the instruments be removed from the patient's body along the axis of the shaft. Whereas conventional instruments (e.g. drills, saws, lasers), can be mounted to the MIRO as shown in Fig. 5 (left), the hollow shaft of axis 7 and the large range of axis 6 allow endoscopic instruments to be mounted as depicted in Fig. 5 (right). Instruments are connected to the end effector of the robot by a failsafe electro-magnetic tool changer, thus allowing for convenient instrument mounting, avoiding difficult-to-clean clamps or bolts and enabling future procedures for autonomous instrument changing.

\section{DLR MICA instrument}

In MIRS, the MICA instruments serve as functional end effectors inside the patient's body, thereby extending the dexterity of the MIRO beyond the skin barrier. These instruments - consisting of a functional end, shaft and motor unit (see Fig. 6) - provide two additional DoFs manipulability and seven DoFs of force/torque sensing inside the body. The functional end of the MICA instrument consists of a two DoF cable-driven universal joint. Together with the MIRO wrist rotation (joint 7), this joint forms a spherical wrist with intersecting axes. Combined with the four DoF intracorporeal motion provided by the MIRO, this enables six DoF manipulability, thus achieving a high level of dexterity $[14,15]$. An additional, actuated, functional DoF allows tissue manipulation. Presently, a straightforward design of the functional DoF is realised in the shape of a gripper for demonstration purposes only. Nevertheless, this multipurpose instrument is capable of gripping tissue, suture material, and even surgical needles bringing them to an upright position when gripping. However, a large variety of customised surgical instruments (e.g. scissors, needle holders, mono- and bipolar HF instruments), which differ mainly in the shape of the jaws, can easily be derived from this generic design. In the first stage, three different endoscopic instruments are in the development stage: Metzenbaum scissors, a Maryland dissector, and a conventional needle holder. This group of instruments is intended to constitute a basic sufficient set to perform simple intracorporeal viscerosynthesis with force feedback for 

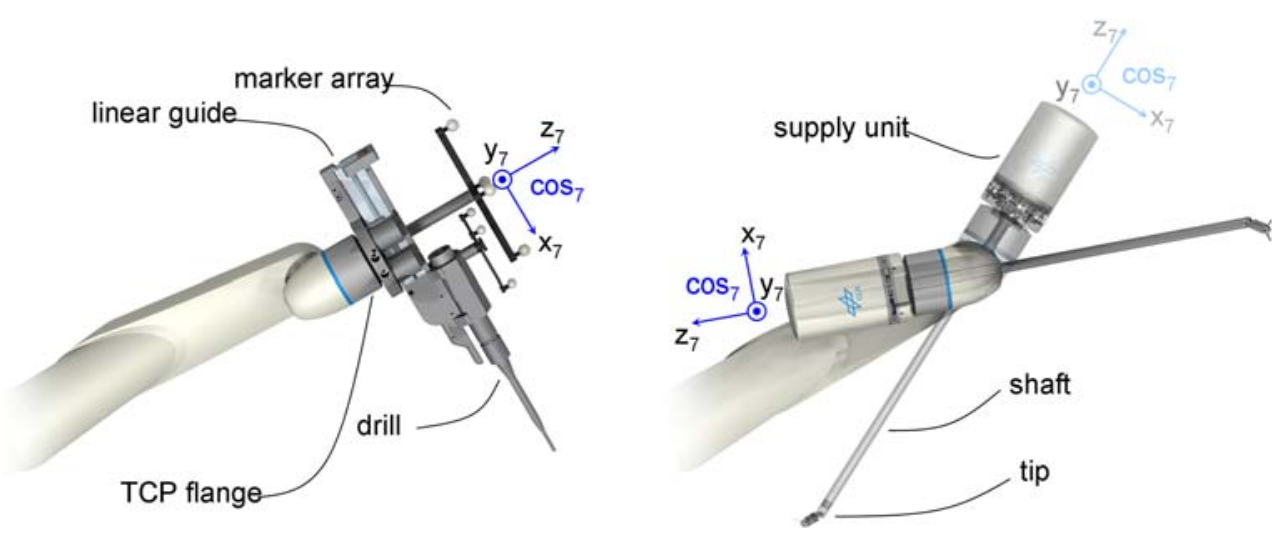

Fig. 5 The DLR MIRO wrist design: configuration for conventional instruments (left) and for endoscopic instruments (right)

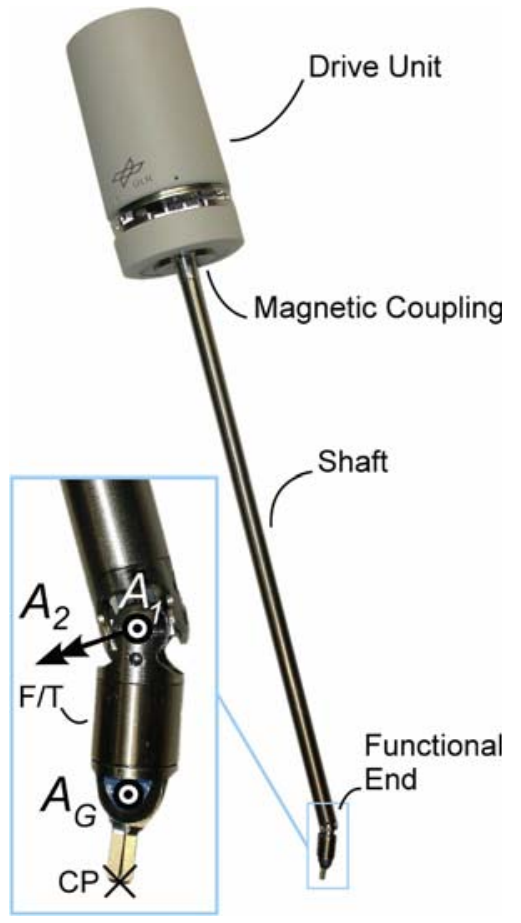

Fig. 6 DLR MICA instrument for endoscopic surgery

further test purposes. The basic specifications of the MICA instruments are shown in Table 2.

Force Feedback in MIRS can reduce the magnitude of forces applied to the tissue and, therefore, reduce unintended punctures and scratches [16]. The integrated Stewart platform, which is the structure a six DoF force/torque sensor, enables the measurement of instrument/tissue interaction forces. Here, the six connecting links between the functional end and the two DoF joint (see Fig. 6) are subjected to stress from tissue interactions. The induced strain is measured by resistive strain-gauges in a half-bridge configuration [15]. Due to the sensor position, the measurement data are distorted neither by friction in the trocar nor by joint actuation
Table 2 Specifications of the DLR MICA instrument

\begin{tabular}{ll}
\hline Parameter & Values \\
\hline Number of joints & $2\left(A_{1}, A_{2}\right)$ \\
Angle range & $\pm 40^{\circ}$ \\
Force range at Tool tip & $\pm 10 \mathrm{~N}$ \\
Functional DoFs & $1\left(A_{\mathrm{G}}\right)$ \\
Maximum grasping force & $40 \mathrm{~N}$ \\
Force/torque sensor & $6 \mathrm{DoFs}$ (manipulation), \\
& $1 \mathrm{DoF}$ (grasping) \\
Force accuracy & $0.04 \mathrm{~N}$ \\
Communication interface & Serial \\
Dimensions motor unit & $\varnothing 65 \times 132 \mathrm{~mm}$ \\
Mass & $0.6 \mathrm{~kg}$ \\
\hline
\end{tabular}

forces and the data can be relayed back to the surgeon's hands to re-establish haptic perception. The sensor has a diameter of $10 \mathrm{~mm}$ and its annular cross section allows for gripper mechanics and an additional one DoF gripping force sensor to be tightly integrated. The parasitic influence of gripper actuation forces on the tissue interaction forces is reduced by careful mechanical layout of the gripper drive train. Electrical signals of both sensors are amplified in close proximity to the sensors by signal conditioning electronics that are integrated in the instrument shaft. Knot tying forces [17], secure needle grasping and various tissue manipulation data [1822] have been used to provide design requirements for sensors and actuation of the instruments. For repeated use, the instruments have to be sterilised by a standard method such as autoclaving. Therefore, each instrument is designed to be separable in two parts: the distal part, which is in direct patient contact, is free of thermo- or humidity-sensitive components and can be autoclaved. The proximal motor unit of the instrument is not in direct patient contact and can simply be disinfected at ambient conditions. It follows modular design principles by integrating motors, absolute and relative position sensors and all the necessary power and communication 
electronics. It can also be easily adapted for different surgical instruments.

\section{Surgical workstation}

During an operation, the surgical workstation is the interface between the surgeon and the system. It provides multimodal and broadband communication to the surgeon in an intuitive and versatile manner. Two different types of user interfaces are being explored with the MiroSurge robotic system. The first one uses haptic interfaces that feed back manipulation forces to the surgeon's hands and displays 3D-vision on a screen. Although the direct force reflection is desirable, a drawback of this approach is the perceptible inertia and friction of haptic devices. An alternative, contact-free approach integrates an interface, which is not based on a kinematic mechanism for position measurements. High level of immersion into the remote environment and an intuitive control for the surgeon are expected of any interface.

\section{Haptic interface}

The haptic hand controllers (Omega.7, by Force Dimension) integrated into the MiroSurge system offer six DoF manipulability and an additional grasping DoF. The translational and the grasping DoFs are actuated and can, therefore, provide force feedback, whereas the rotatory DoFs are passive and equipped with encoders. Bimanual teleoperation is realised with dedicated left- and right-handed devices, as shown in Fig. 7. Motions of the haptic devices between the surgeon's fingers are mapped onto the virtual control point (cp) at the MICA instruments' tips as shown in Fig. 6. The surgeon controls the tips of the instruments in the same way he controls his own hands and feels the contact forces. The grasping is independently controlled with the forefinger. The position

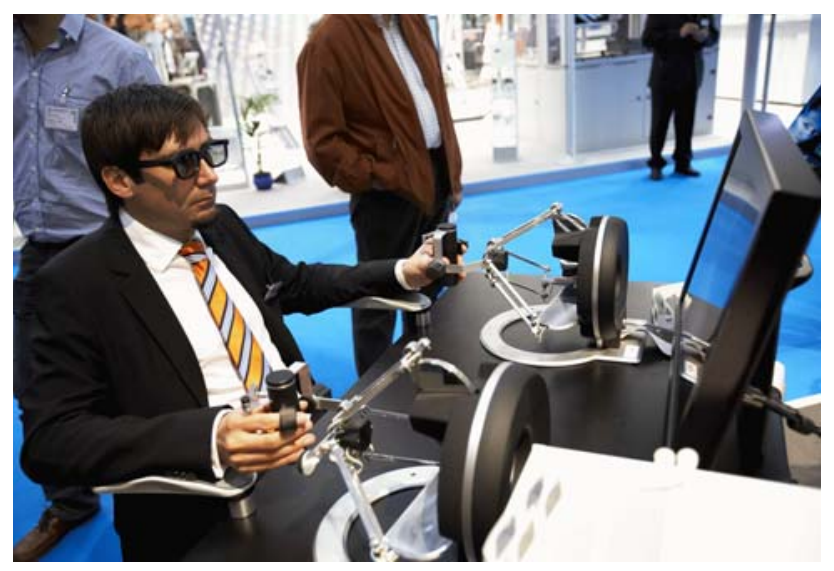

Fig. 7 The MiroSurge user interface: configuration with two Omega.7 devices and Miracube display

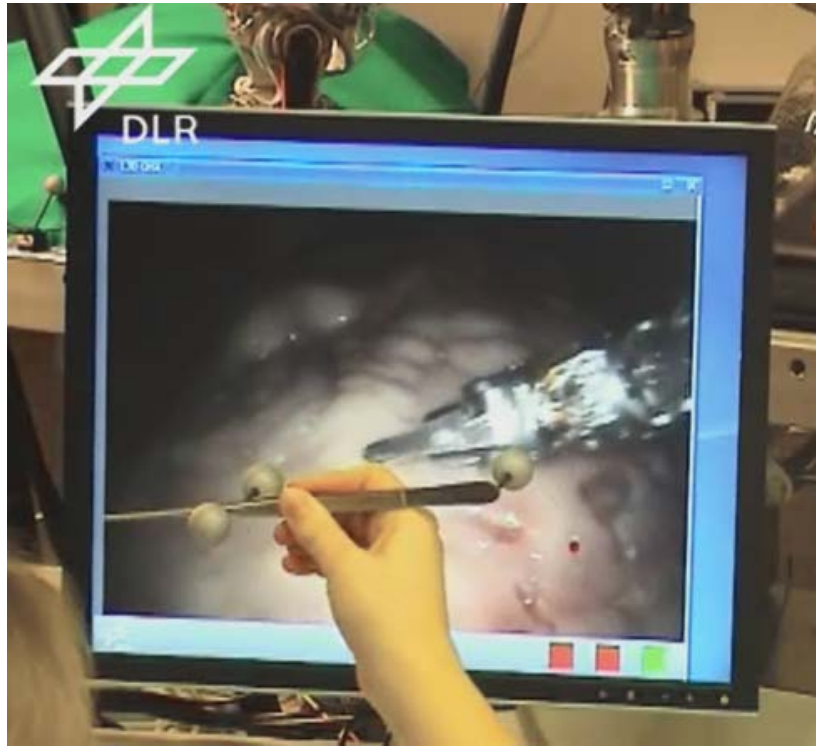

Fig. 8 The MiroSurge user interface: tracking of conventional instruments

of the endoscope can be controlled through the same interface. Adaptation of workspaces is done by indexing using a foot pedal.

In experiments with a Freedom 7S (MPB Technologies Inc.), feedback of manipulation torques was also realised.

\section{Contact-free interface}

A second way of controlling the system is implemented through the use of standard surgical forceps equipped with reflecting markers, as depicted in Fig. 8. The markers are tracked by an optical measurement system (SmARTtrack, A.R.T. $\mathrm{GmbH}$ ), and the tip of the hand-held instrument is mapped onto the control point (cp) of the MICA instrument inside the patient. In this way, the surgeon can use familiar instruments with low inertia to control the robotic system. Since direct force feedback is not possible with this approach, forces are displayed in the form of virtual arrows which augment the stereo vision stream.

\section{Vision}

Visual data from the endoscopic camera have to be available on different systems in parallel. Among these are displays for the surgeon and operating room staff, visual servoing systems, and documentation systems. Therefore, the MiroSurge system provides multicast functions to distribute the endoscopic stereo video to various displays. The stereo image stream is captured by a video server at a frame rate of $25 \mathrm{~Hz}$ and an image resolution of $768 \times 576(\mathrm{PAL})$ for the left and right images. The image stream is augmented with information about measured interaction forces and distrib- 


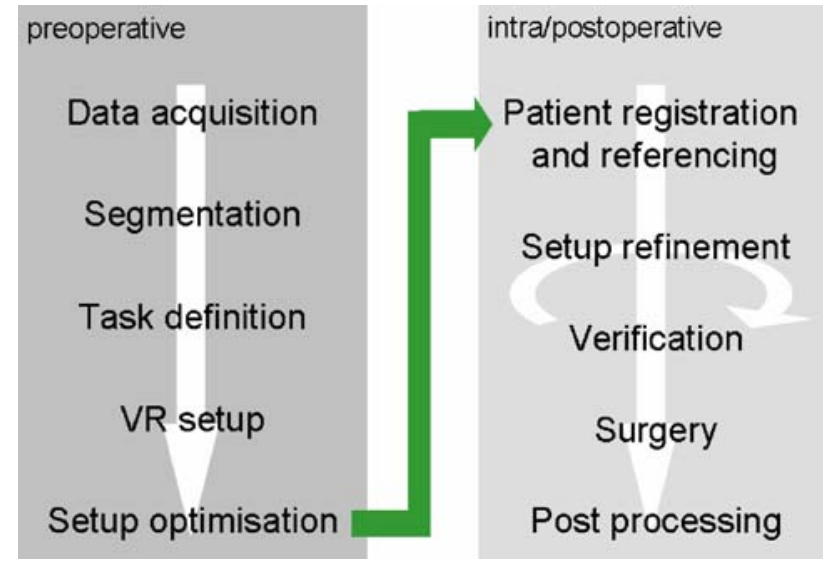

Fig. 9 The MiroSurge planning and setup procedure

uted to a flexible number of clients. The main display is directly fed by the video server via shared memory communication, reducing latency to a minimum. Further clients can be connected via Ethernet, which involves some additional latency for video stream coding and transmission $(<40 \mathrm{~ms})$. The configuration shown in Fig. 1 integrates an autostereoscopic screen (SeeFront $\mathrm{GmbH}$ ), based on a fixed lenticular array, with eye tracking to process and display left and right images. Alternatively, or as a supplement, a polarisation-based stereo display (Miracube) can be integrated as shown in Fig. 7. Moreover, the stereoscopic image stream can also be used to implement vision-based control applications, e.g. instrument tracking for automated camera guidance [23] or tracking of organ movements for motion compensation [24].

\section{Planning and registration}

In addition to standard surgery planning (choosing a treatment plan according to the diagnosis), the use of a robotic system in the operating room (OR) requires further planning to optimise the robotic setup. This includes determining positions for the robots in the OR to optimise several criteria. For example, no collisions between robots should occur, but the entire operating field must be accessible by all robots. Singularities and joint limits must also be avoided. Once an optimal setup is planned, an easily executable setup and registration procedure is crucial to speed up and control the setup in the OR.

Hence, a comprehensive setup procedure, which is valid for a wide range of robotically assisted applications, was developed [25]. Its implementation allows for a variable number of robots of different types to be mounted in a variety of configurations. The scheme of the procedure is shown in Fig. 9 and is exemplified by the MiroSurge telesurgery application.
Data acquisition (e.g. CT scan) is often used during standard surgery planning and can be directly adopted for use with the robotic system. Segmentation involves labelling the relevant structures of the patient and facilitates subsequent task definition. Several tools, such as amira ${ }^{\circledR}$ by Mercury Computer Systems, are available for segmentation. However, segmentation is not mandatory for the described workflow. During task definition, the relevant operating volume is identified (see Fig. 10, right), and an area for possible entry points is defined. In the virtual reality $(V R)$ setup, the digitised patient is positioned on the virtual OR table according to the treatment plan. The number and coarse arrangement of the robots are then determined, possibly with the use of templates derived from previous operations, and the search space (see Fig. 10, left) is defined.

Setup optimisation is then carried out automatically using genetic algorithms and a subsequent gradient-based method. To rank different setups of the robotic system, the algorithm simulates the motions of all robots, including various relevant tip rotations, inside the discrete operating volume. It evaluates criteria such as reachability of the entire operating volume, manipulability, accuracy, and collision probability. Since fixed paths cannot be predetermined in telesurgery applications, all combinations of robot tip positions inside the operating volume are considered for collision detection. As a result, diverse feasible setups are proposed. The surgeon then chooses the best one according to his preferences and expert knowledge.

The previously described steps are usually done preoperatively. Once inside the operating room, patient registration and referencing, with respect to the OR table, are performed using a surface-based method. The DLR 3D modeller's triangulation sensor (light stripe profiling) [26] is used as the optical distance sensor. A streaming surface reconstruction method generates online the corresponding mesh. The developed registration algorithm combines a feature-based method with the standard iterative closest point method. The featurebased method is a modified version of the Barequet algorithm [27] with additional surface normal information. The scan of the patient as well as the registration algorithm take only several seconds. The algorithm does not require initial manual alignment and is therefore fully automatic. A subsequent refinement of the optimisation is necessary to take into account the current situation in the real OR. Optimal positions for the robots and their entry points are then automatically displayed using a hand-held laser projector, and the robots can be mounted to the rails of the OR table as specified. In the verification step, the robots are positioned in the "approach pose," where each robot points towards its entry point into the patient. This allows for visual inspection and approval of the setup, before the operation begins. During the operation, the VR is permanently updated with sensor data from the real robotic system (e.g. joint angles of 


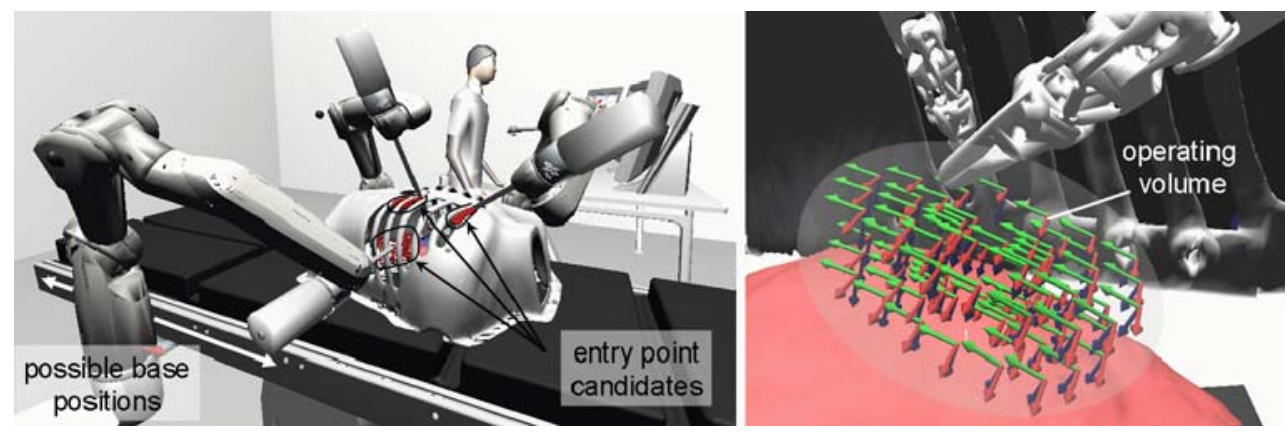

Fig. 10 Task definition and VR setup: The operating volume inside the patient (right) must be reachable while certain criteria are optimised. The search space consists of the robot base positions and entry points (left)

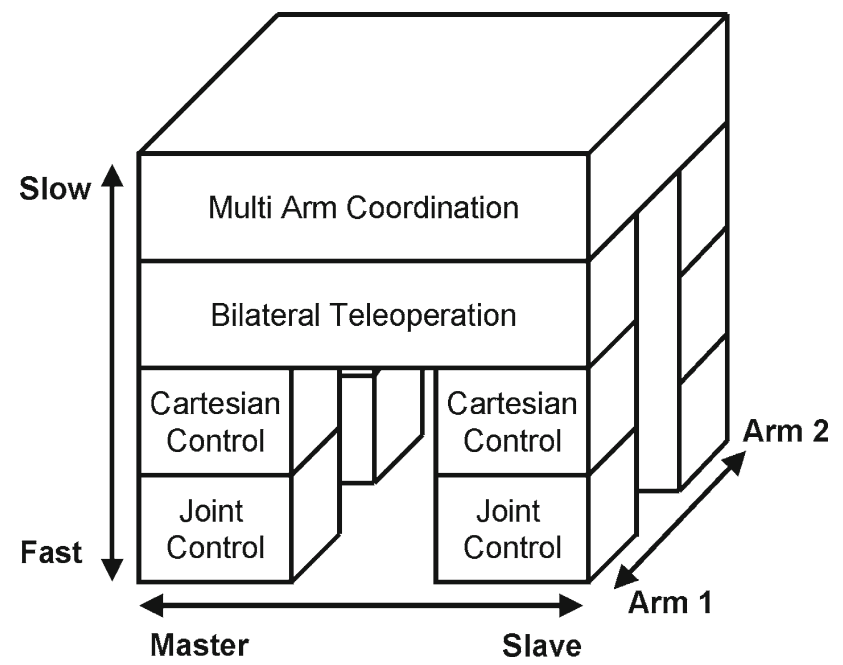

Fig. 11 The MiroSurge four layer architecture in three dimensions

the robots). This allows for further monitoring of the procedure. Post processing consists of storing and evaluating the setup and procedure data to provide new templates for later interventions.

\section{Control of the telerobotic system}

The robotic system's control software has to integrate a workflow, handle various control loops and feed forward control signals. It has to be efficiently executed and should be flexible for rapidly prototyping new features and applications.

\section{Control architecture}

The telerobotic system has a hierarchical structure with four major control layers, as shown in Fig. 11. This architecture aims to satisfy two major goals: (a) structuring of software components according to their demands in execution time.

(b) Development of software and control components within a group of developers using different levels of abstraction.
On higher levels, more hardware is included, but more details are abstracted.

- Layer 1 (joint control): This layer controls the joint positions and torques of a robot.

- Layer 2 (local Cartesian control): In this layer, the complete mechanical chains, with all joints, kinematics and dynamics, are considered.

- Layer 3 (bilateral teleoperation): This layer connects two Cartesian devices to a one arm master-slave system for bilateral teleoperation.

- Layer 4 (multi-arm coordination): Two master-slave systems for the left and the right hand of the surgeon are integrated into a bimanual teleoperation system. The endoscopic camera is connected to this layer. In general all components that demand neither high rates $(>200 \mathrm{~Hz})$ nor low latencies are located here.

The following sections describe the MIRO control modes for position and compliant control on the joint level (layer 1), Cartesian level (layer 2), and bilateral teleoperation control level (layer 3). A short description is given below on the implementation of the control software.

\section{MIRO control}

The MIRO has two main control modes: one is the classic position control mode, in which the robot follows a desired trajectory for accurate positioning. The other is a compliant control mode, i.e. the soft robotics approach. Both can be divided into basic functionalities on the joint level with Cartesian extensions.

In the position control mode the robot tracks a commanded link side position. The elasticity of the robot due to the lightweight concept is taken into account with a flexible joint model. The commanded link side positions are transformed into desired motor positions and desired torques. A state feedback controller computes these variables, with the measured states being given by the motor positions, the joint torques 
and the calculated derivatives of these positions and torques [28]. This controller provides an effective vibration damping.

In the telerobotic scenario, the joint position control mode is used with an interpolator to move the robot from its initial configuration to the "approach pose," which is close to the entry points of the patient. Since this controller works in joint space, an inverse kinematics that transforms Cartesian positions and orientations into joint space is used to allow Cartesian position commands. The inverse kinematics is calculated analytically to get the first valid solution. A numerical algorithm iteratively optimises the nullspace configuration with respect to several criteria, such as avoidance of joint limits, singularities and collisions.

In the compliant mode, the user can guide the robot on a desired trajectory by exerting manual forces on the robot's structure. A torque controller effectively reduces motor masses and friction felt by the user and provides the torques needed to counter gravity and hold the robot's weight [13,29]. Cartesian compliances can be imposed on the robot's tip, while the elbow can still be moved freely in the nullspace. Several virtual springs are attached to the tip so that the robot follows a desired trajectory in free motion or exerts a certain force on the surrounding environment. Stiffness and damping can be adjusted separately in all six Cartesian DoFs. This is used, e.g. for the manual insertion of endoscopic instruments into the patient. First, the rotations are practically locked due to high stiffness, and three-DoF translational guidance is enabled for the user. Once the instrument enters the trocar, two more DoFs are physically restricted and motion is allowed only along the direction of the shaft.

\section{Teleoperation}

Bilateral teleoperation is based on a position-force architecture with the haptic input device (master) sending positions to and receiving interaction forces from the robot and the instrument (slave). Poses of the master device are rotated so that the haptic channel is aligned with the visual channel to ensure proper hand-eye-coordination. The inverse kinematics of the MIRO is expanded so that all the joint angles of the MIRO and the MICA can be calculated. The system automatically decouples when the slave's limits are reached and recouples when the user moves back into the workspace. Workspace limitations of the telemanipulator can be defined in either joint space or Cartesian space as user-defined restricted areas. This interface handles all workspace limitations on the slave and allows convenient integration of different user interfaces.

The motion-scaling factor $s_{\text {velocity }}$ between master and slave can be preset arbitrarily by the user with the control interface of the MiroSurge system. A scaling factor smaller than 1 means that the instruments move with reduced velocity, which reduces non-linearities and noise in the haptic devices as well as unintended user motion (tremor). However, if the scaling is too small the workspace limits of the haptic devices are reached and greater motions of the user lead to fatigue. Since an optimal scaling depends on the tasks in the operation, the duration and the user, this parameter can be set by the user and the parameter for the bilateral teleoperation are adapted to generate a force feedback corresponding to the motion scaling and to ensure stability of the system. Tests show that in order to ensure stability in all conditions the forces should not be amplified with more than $s_{\text {force }}=\frac{1}{s_{\text {velocity }}} \cdot \frac{1}{3}$. For performing a single suture a scaling factor of $0.25 \leq s_{\text {velocity }} \leq 0.5$ has been identified as a good compromise balancing the above-mentioned influences.

With the position-force architecture, the user does not feel forces coming from the slave when moving in free space. However, the user can clearly distinguish between hard and soft contacts such as instrument collisions or tissue interactions.

\section{Distributed control}

The control software was developed with Matlab/Simulink and the code was automatically generated using Real Time Workshop. The compiled code runs on a QNX Neutrino realtime operating system and is interfaced with Matlab/Simulink external mode for development and debugging. The executables are distributed on six off-the-shelf PCs with $Q N X$ (see Fig. 12). Interprocess communication is implemented with aRDnet (agile Robot Development) [30], as a decentralised net of blocks communicating over I/O-ports on a local network. Local joint controllers for the MIROs are running at a $3-\mathrm{kHz}$ rate, which is synchronised on the robot's sensors. The models implementing the force feedback are executed at a 1$\mathrm{kHz}$ rate. The bottleneck in the current setup is the serial communication with the MICA, where a 4-ms sampling time and asynchronous connections exist. The latency from the F/Tsensor to the haptic device and back to the MICA's motors can be up to $10 \mathrm{~ms}$. The software is expandable and flexible for research in a rapid prototyping environment.

\section{Conclusions}

This paper presents the new endoscopic telesurgery system, MiroSurge. On the patient side, MIRO robots hold an endoscope and dedicated instruments. The instruments enable dexterous motion and force sensing inside the patient. The surgical workstation is comprised of various displays and input devices with or without force feedback. The system is configurable with respect to the number of robots and their setup (wall, ceiling, or table mounted). The components of the MiroSurge system were shown to fulfil the rigid design requirements for robotic telesurgery with force feed- 


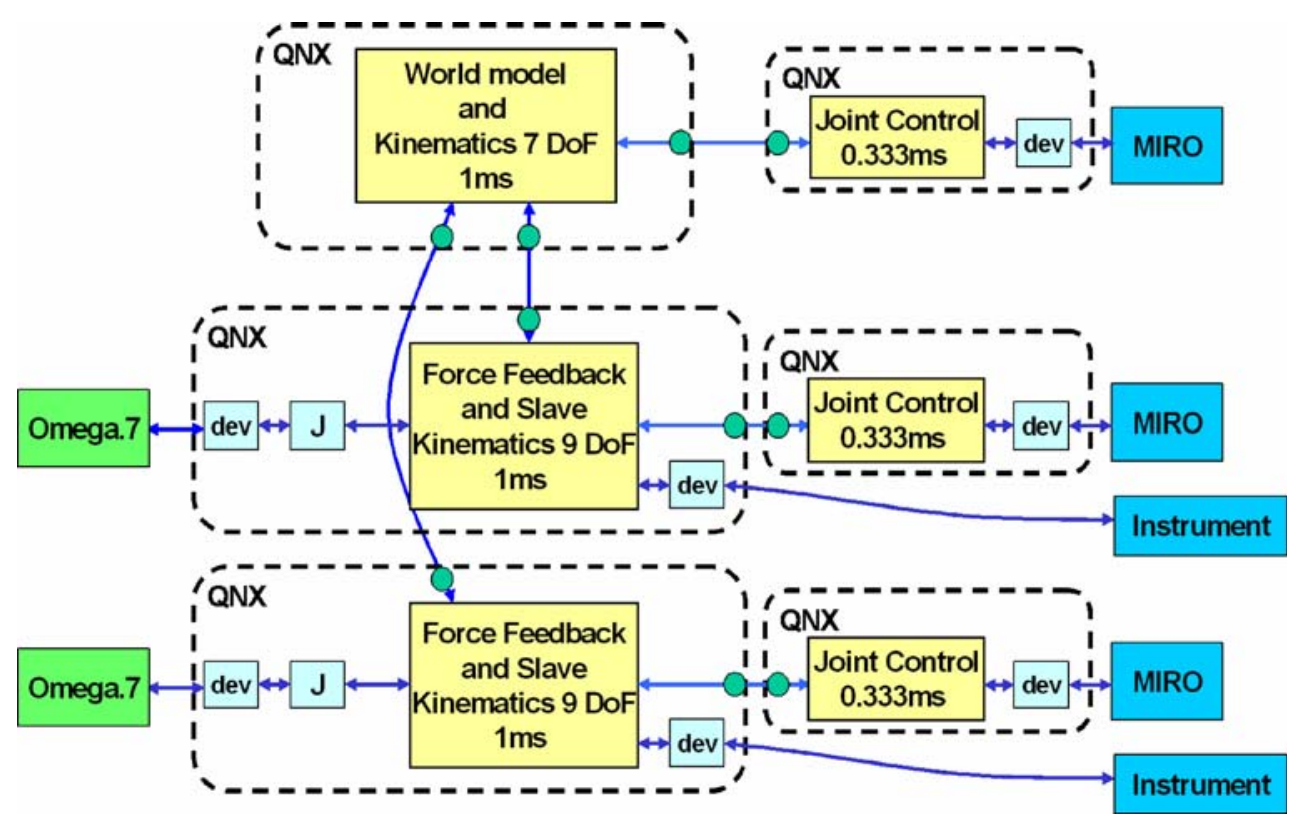

Fig. 12 MiroSurge: The current implementation in a distributed system with six off-the-shelf PCs

back $[9,14,25]$. This paper presents the integration of those components. As a result, the MiroSurge system allows for surgical tasks, such as knot tying, with force feedback that can discern soft from hard contacts. At the same time, the system remains versatile, a key feature for further system development. It allows for rapid prototyping of new features as well as adapting and optimising the system for close contact with surgeons.

\section{Outlook}

Besides testing and improving the DLR MiroSurge telerobotic platform according to international standards (e.g.; EN61508, IEC60601-1, 3. edition), ongoing research projects are focusing on the following approaches:

New sensor technologies, such as ultrasound, are being integrated for various tasks including detecting hidden arteries beneath covering tissue. By modifying the jaws of the DLR MICA a Metzenbaum scissor, Maryland dissector, and a conventional needle holder are under development.

Beating heart motion is being compensated to facilitate complicated tasks in cardiac surgery. This compensation is assumed to also benefit the patient by avoiding the use of a heart lung machine. Compensation is achieved by detecting motion from endoscopic images by tracking natural landmarks on the surface of the beating heart [24,31]. These data are then fed back to the robot system $[32,33]$ and the instruments are moved in synchrony with the beating heart. A stabilised 3D image of the scene is also in development. Using this feature, the surgeon can operate on a virtually still standing surface of the heart.
Furthermore, the setup for robot-controlled laser osteotomies is currently being developed to equip a single MIRO robot for use in orthopaedic surgery.

Acknowledgments We gratefully acknowledge the Bavarian Research Foundation (BFS) for funding the MiroSurge project. Special thanks to Paul Mittermiller.

\section{References}

1. Hagn U, Nickl M, Jörg S, Tobergte A, Kübler B, Passig G, Gröger M, Fröhlich F, Seibold U, Konietschke R, Le-Tien L, AlbuSchäffer A, Grebenstein M, Ortmaier T, Hirzinger G (2008) DLR MIROSURGE - towards versatility in surgical robotics. In: Proceedings of CURAC 2008, Leipzig, pp 143-146

2. Green PS, Hill JW, Jensen JF, Shah A (1995) Telepresence surgery. IEEE Eng Med Biol 14(3): 324-329. doi:10.1109/51.391769

3. Phee L, Xiao D, Yuen J, Chan CF, Ho H, Thng CH, Cheng C, $\mathrm{Ng}$ WS (2005) Ultrasound guided robotic system for transperineal biopsy of the prostate. In: Proceedings of the IEEE international conference on robotics and automation (ICRA) 2005, Barcelona, pp 1315-1320

4. Smith-Guerin N, Nouaille L, Vievres P, Poisson G (2008) A medical robot kinematics design approach based on knowledge management. Ind Robot 35(4): 316-323. doi:10.1108/ 01439910810876418

5. Albers J, Schmidt T, Hassfeld S, Heid F, Vahl C (2007) Sternotomy and craniotomy by an autonomous robot: experimental evaluation on seven pigs. Zeitschrift für Herz-, Thorax- und Gefäßchirurgie. doi:10.1007/s00398-007-0599-8

6. Ortmaier T, Weiß H, Hagn U, Nickl M, Albu-Schäffer A, Ott C, Jörg S, Konietschke R, Le-Tien L, Hirzinger G (2006) A handson-robot for accurate placement of pedicle screws. In: Proceedings of the IEEE international conference on robotics and automation (ICRA) 2006, Orlando, pp 4179-4186 
7. Döbele S (2008) Die transpedikuläre Bohrung: eine biomechanische und theoretische Analyse der transpedikulären Bohrung zur Auslegung eines navigationsgestützten, semi-aktiven Roboters. $\mathrm{PhD}$ thesis, Technische Universität München, Munich, Germany

8. Konietschke R, Ortmaier T, Ott C, Hagn U, Le-Tien L, Hirzinger G (2006) Concepts of human-robot co-operation for a new medical robot. In: Proceedings of the second international workshop on human centered robotic systems (HCRS) 2006, Munich, pp 1-6

9. Hagn U, Nickl M, Jörg S, Passig G, Bahls T, Nothhelfer A, Hacker F, Le-Tien L, Albu-Schäffer A, Konietschke R, Grebenstein M, Warpup R, Haslinger R, Frommberger M, Hirzinger G (2008) The DLR MIRO - a versatile lightweight robot for surgical applications. Ind Robot 35(4). doi:10.1108/01439910810876427

10. Konietschke R, Ortmaier T, Weiss H, Hirzinger G (2004) Manipulability and accuracy measures for a medical robot in minimally invasive surgery. In: Lenarcic J, Galletti C (eds) On advances in robot kinematics. Kluwer, New York pp 191-198

11. Frumento S, Michelini R, Konietschke R, Hagn U, Ortmaier T, Hirzinger G (2006) A co-robotic positioning device for carrying surgical end-effectors. In: Proceedings of ASME-ESDA 2006, Torino, pp 1-8

12. Haddadin S, Albu-Schäffer A, Hirzinger G (2007) Safety evaluation of physical human-robot interaction via crash-testing. In: Proceedings of RSS2007, Atlanta, pp $217-224$

13. Albu-Schäffer A, Ott C, Hirzinger G (2007) A unified passivity based control framework for position, torque and impedance control of flexible joint robots. Int J Robot Res 26(1): 23-39. doi:10. $1177 / 0278364907073776$

14. Kuebler B, Seibold U, Hirzinger G (2005) Development of actuated and sensor integrated forceps for minimally invasive robotic surgery. Int J Med Robot Comput Surg. doi:10.1581/mrcas.2005. 010305 and $10.1002 / \mathrm{rcs} .33$

15. Seibold U, Kuebler B, Hirzinger G (2008) Prototypic force feedback instrument for minimally invasive robotic surgery. In: Bozovic V (ed) Medical robotics. I-Tech Education and Publishing, Vienna pp 377-400

16. Wagner C, Stylopoulos N, Howe R (2002) The role of force feedback in surgery: analysis of blunt dissection. In: Proceedings of the 10th symposium on haptic interfaces for virtual environment and teleoperator systems (HAPTICS), pp 68-74, ISBN:0-7695-1489-8

17. Kitagawa M, Dokko D, Okamura AM, Bethea BT, Yuh DD (2004) Effect of sensory substitution on suture manipulation forces for surgical teleoperation. J Thorac Cardiovasc Surg 2004:151-158

18. Abolhassani N, Patel R, Moallem M (2007) Needle insertion into soft tissue: a survey. Med Eng Phys 29(4). doi:10.1016/j. medengphy.2006.07.003

19. Frick TB, Marucci DD, Cartmill JA, Martin CJ, Walsh WR (2001) Resistance forces acting on suture needles. J Biomech 34(10): 1335-1340. doi:10.1016/S0021-9290(01)00099-9

20. Maurin B, Barbe L, Bayle B, Zanne P, Gangloff J, de Mathelin M, Gangi A, Soler L, Forgione A (2004) In-vivo study of forces during needle insertions. In: Buzug TM, Lueth TC (eds) Perspectives in image-guided surgery, World Scientific Pub Co, Singapore, pp 415-422
21. Okamura AM, Simone C, O’Leary MD (2004) Force modeling for needle insertion into soft tissue. IEEE Trans Biomed Eng 51(10). doi:10.1109/TBME.2004.831542

22. Podder TK, Sherman J, Clark DP, Messing EM, Rubens DJ, Strang JG, Liao L, Brasacchio RA, Zhang Y, Ng WS, Yu Y (2005) Evaluation of robotic needle insertion in conjunction with in vivo manual insertion in the operating room. In: Proceedings of the IEEE Roman 2005. doi:10.1109/ROMAN.2005.1513758

23. Wei GQ, Arbter K, Hirzinger G (1997) Real-time visual servoing for laparoscopic surgery. IEEE Eng Med Biol 16(1):40-45. doi:10. $1109 / 51.566151$

24. Groeger M, Ortmaier T, Sepp W, Hirzinger G (2002) Tracking local motion on the beating heart. Proc SPIE 4681:233. doi:10.1117/12. 466926

25. Konietschke R (2007) Planning of Workplaces with Multiple Kinematically Redundant Robots. Ph.D. thesis, Technische Universität München, Germany

26. Suppa M, Kielhofer S, Langwald J, Hacker F, Strobl KH, Hirzinger G (2007) The 3D-modeller: a multi-purpose vision platform. In: Proceedings of the IEEE international conference on robotics and automation (ICRA) 2007, Rome, doi:10.1109/ROBOT.2007. 363081

27. Barequet G, Sharir M (1997) Partial surface and volume matching in three dimensions. IEEE Trans Pattern Anal Mach Intell(T-PAMI) 19(9):929-948

28. Le-Tien L, Albu-Schäffer A, Hirzinger G (2007) MIMO state feedback controller for a flexible joint robot with strong joint coupling. In: Proceedings of the IEEE international conference on robotics and automation (ICRA) 2007, Rome, doi:10.1109/ROBOT.2007. 364065

29. Ott C, Albu-Schäffer A, Hirzinger G (2004) A passivity based Cartesian impedance controller for flexible joint robots-Part I: torque feedback and gravity compensation. In: Proceedings of the IEEE international conference on robotics and automation (ICRA) 2004, New Orleans, pp 2659-2665

30. Baeuml B, Hirzinger G (2006) Agile robot development (aRD): a pragmatic approach to robotic software. In: Proceedings of the IEEE international conference on intelligent robots and systems (IROS) 2006, Beijing, doi:10.1109/IROS.2006.281756

31. Groeger M, Sepp W, Hirzinger G (2005) Structure driven substitution of specular reflections for realtime heart surface tracking. In: Proceedings of the IEEE international conference on image processing (ICIP) 2005, Genova, 2, pp 1066-1069

32. Ortmaier $\mathrm{T}$, Groeger $\mathrm{M}$, Boehm DH, Falk V, Hirzinger G (2005) Motion estimation in beating heart surgery. Ieee Trans Biomed Eng 52(10):1729-1740

33. Groeger M, Hirzinger G (2006) Image stabilisation of the beating heart by local linear interpolation. Proc SPIE 6141:61410X. doi:10. $1117 / 12.654119$ 